\title{
Training Deep Neural Networks in Generations: A More Tolerant Teacher Educates Better Students
}

\author{
Chenglin Yang, Lingxi Xie, ${ }^{\circledR}$ Siyuan Qiao, Alan L. Yuille \\ Johns Hopkins University \\ \{chenglin.yangw,198808xc,joe.siyuan.qiao,alan.l.yuille\}@ gmail.com
}

\begin{abstract}
We focus on the problem of training a deep neural network in generations. The flowchart is that, in order to optimize the target network (student), another network (teacher) with the same architecture is first trained, and used to provide part of supervision signals in the next stage. While this strategy leads to a higher accuracy, many aspects (e.g., why teacher-student optimization helps) still need further explorations.

This paper studies this problem from a perspective of controlling the strictness in training the teacher network. Existing approaches mostly used a hard distribution (e.g., one-hot vectors) in training, leading to a strict teacher which itself has a high accuracy, but we argue that the teacher needs to be more tolerant, although this often implies a lower accuracy. The implementation is very easy, with merely an extra loss term added to the teacher network, facilitating a few secondary classes to emerge and complement to the primary class. Consequently, the teacher provides a milder supervision signal (a less peaked distribution), and makes it possible for the student to learn from inter-class similarity and potentially lower the risk of over-fitting. Experiments are performed on standard image classification tasks (CIFAR100 and ILSVRC2012). Although the teacher network behaves less powerful, the students show a persistent ability growth and eventually achieve higher classification accuracies than other competitors. Model ensemble and transfer feature extraction also verify the effectiveness of our approach.
\end{abstract}

\section{Introduction}

\section{- "Indigo comes from blue, but it is bluer than blue."} -AN OLD PROVERB

Deep learning, especially the convolutional neural networks, has been widely applied to computer vision problems. Among them, image classification has been considered the fundamental task which sets the backbones vision systems for other problems (Krizhevsky, Sutskever, and Hinton 2012)(Simonyan and Zisserman 2015)(Szegedy et al. 2015)(He et al. 2016), and the knowledge or features extracted from these modules are transferrable for generic image representation purposes (Razavian et al. 2014) or other vision tasks (Long, Shelhamer, and Darrell 2015)(Ren et al. 2015)(Xie and Tu 2015)(Newell, Yang, and Deng 2016).

Copyright (C) 2019, Association for the Advancement of Artificial Intelligence (www.aaai.org). All rights reserved.
A fundamental task in computer vision is to optimize deep networks for image classification. Most existing work achieved this goal by fitting the outputs of a model to onehot vectors. For each training sample $\left(\mathbf{x}_{n}, y_{n}\right)$ where $\mathbf{x}_{n}$ is an image matrix and $y_{n}$ is the class label (out of $C$ classes), the goal is to find network parameters $\boldsymbol{\theta}$, so that $\mathbf{f}\left(\mathbf{x}_{n} ; \boldsymbol{\theta}\right) \approx[0, \ldots, 1, \ldots, 0]^{\top} \in \mathbb{R}^{C}$, i.e., only the $y_{n}$-th dimension is 1 and all others are 0 . Despite its effectiveness, this is not necessarily the optimal target to fit, because except for maximizing the confidence score of the primary class (i.e., the ground-truth), allowing for some secondary classes (i.e., those visually similar ones to the ground-truth) to be preserved may help to alleviate the risk of over-fitting. Some proposed to learn a class-level similarity matrix (Deng et al. 2010)(Verma et al. 2012)(Wu, Tygert, and LeCun 2017), but these approaches are unable to capture inter-class similarities at the image level, e.g., different cat images may be visually similar to different classes.

We turn to an alternative solution, i.e., extracting knowledge from a trained (teacher) network and guide another (student) network in an individual training process. This algorithm, known as knowledge distillation, was first used to network compression (the student network is much smaller than the teacher network, but can achieve a comparable accuracy) (Hinton, Vinyals, and Dean 2015), but later it was verified effective in the scenario that teacher and student networks have the same architecture, in which the student is expected to achieve a higher accuracy than the teacher (Furlanello et al. 2018). Despite its effectiveness, it remains unclear how teacher-student optimization works, and if we can find some key factors to guide the design of such optimization processes.

This paper provides an interesting perspective, focusing on the strictness of the teacher. We argue that classification accuracy is not the major goal of the teacher network; instead, it is designed to be tolerant (i.e., producing less peaked distributions of confidence) so that the students can learn inter-class similarity and potentially prevent over-fitting. To achieve this goal, we add an extra term to the standard crossentropy loss in training the teacher network, facilitating it to distribute confidence to a few secondary classes. Although this harms the accuracy of the teacher network, it indeed provides more room for the student network(s), and eventually, the students are better than those educated by a strict teacher. 
In standard image classification experiments on CIFAR100 and ILSVRC2012, our approach reports higher classification accuracy than its competitors, regardless using a single model, an ensemble of multiple models, or transferring trained models for feature extraction.

The contribution of this work is three-fold. First, we propose a new perspective to interpret why teacher-student optimization works. Second, we suggest an evaluation method to quantize its impact. Third, we design an efficient "tolerantteacher" framework which achieves superior performance.

The reminder of this paper is organized as follows. We first review related work in the next section, and then illustrate our approach. After experiments are shown, we conclude our work in the final section.

\section{Related Work \\ Deep Learning and Neural Networks}

Deep learning has been dominating the field of computer vision. Powered by large-scale image datasets (Deng et al. 2009) and powerful computational resources, it is possible to train very deep networks for various computer vision tasks. The fundamental idea of deep learning is to design a hierarchical structure containing multiple layers, each of which contains a number of neurons having the same or similar mathematical functions. Researchers believe that a sufficiently deep network is able to fit very complicated distributions in the feature space. In a fundamental task known as image classification, deep neural networks (Krizhevsky, Sutskever, and Hinton 2012) have achieved much higher accuracy than conventional handcrafted features (Perronnin, Sanchez, and Mensink 2010). Towards better recognition performance, researchers designed deeper and deeper networks (Simonyan and Zisserman 2015)(Szegedy et al. 2015)(He et al. 2016)(Huang et al. 2017b)(Hu, Shen, and Sun 2018), and even proposed to automatically explore network architectures (Xie and Yuille 2017)(Zoph and Le 2017).

The rapid progress of deep learning has helped a lot of computer vision tasks. Features extracted from trained classification networks can be transferred to small datasets for image classification (Donahue et al. 2014), retrieval (Razavian et al. 2014) or object detection (Girshick et al. 2014). An even more effective way is to insert specified network modules for these tasks, and initializing these models with part of the weights learned for image classification. This flowchart, often referred to as fine-tuning, works well in a variety of problems, including object detection(Girshick 2015)(Ren et al. 2015), semantic segmentation (Long, Shelhamer, and Darrell 2015)(Chen et al. 2016), edge detection (Xie and Tu 2015), etc.

\section{Training Very Deep Networks in Generations}

Deep network optimization has become an important yet challenging problem. Training very deep neural networks (e.g., more than 100 layers) requires specifically designed techniques to assist numerical stability, such as ReLU activation (Nair and Hinton 2010), Dropout (Srivastava et al. 2014) and batch normalization (Ioffe and Szegedy 2015).
However, as depth increases, the large number of parameters makes neural networks easy to be over-fitted, especially when the amount of training data is limited. Therefore, it is often instructive to introduce extra priors to constrain the training process and thus prevent over-fitting. A common prior assumes that some classes are visually or semantically similar (Deng et al. 2010), and adds a class-level similarity matrix to the loss function (Verma et al. 2012)(Wu, Tygert, and LeCun 2017), but it is unable to deal with per-image similarity which is well noted in previous research (Wang et al. 2014)(Akata et al. 2016)(Zhang, Cheng, and Tian 2018).

An effective way to solve this issue is teacher-student optimization, in which a teacher network is trained beforehand, and then used to guide the optimization of a student network. Thus, the output of the teacher network carries class-level similarity in its confidence score for each image. Previously, teacher-student optimization was used to distill knowledge from a larger network and then compress it into a smaller network (Hinton, Vinyals, and Dean 2015), or initialize a deeper network with pre-trained weights of a shallower network (Romero et al. 2014)(Chen, Goodfellow, and Shlens 2015)(Simonyan and Zisserman 2015). This basic idea was then extended in many ways, including using various ways of supervision (Szegedy et al. 2016)(Pereyra et al. 2017), using multiple teachers to provide a better guidance (Tarvainen and Valpola 2017), adding supervision in intermediate neural responses (Yim et al. 2017), and allowing two networks to help optimize each other (Zhang et al. 2017c). In a recent work named the born-again network (Furlanello et al. 2018), this method was used to optimize the same network in generations, in which the next generation was guided by two terms, namely, the standard cross-entropy loss and the KL-divergence between the teacher and student signals.

\section{Our Approach}

This section presents our approach. We first introduce a framework of optimizing neural networks in generations, and then provide an empirical analysis on the benefits of such optimization methods, and suggest a quantitative way of evaluating this process. Based on these, we finally design a flowchart, which train a tolerant teacher network to improve the overall performance of optimization.

\section{Teacher-Student Optimization}

We consider a standard network optimization task. Given a model $\mathbb{M}$ which has a parameterized form of $\mathbf{y}=\mathbf{f}(\mathbf{x} ; \boldsymbol{\theta})$, where $\mathbf{x}$ and $\mathbf{y}$ are input and output, and $\boldsymbol{\theta}$ denotes learnable parameters (e.g., convolutional weights). Given a training set $\mathcal{D}=\left\{\left(\mathbf{x}_{1}, \mathbf{y}_{1}\right), \ldots,\left(\mathbf{x}_{N}, \mathbf{y}_{N}\right)\right\}$, the goal is to determine the parameter $\boldsymbol{\theta}$ that best fits these data.

One of the most popular optimization methods starts with setting all weights as random noise $\boldsymbol{\theta}^{(0)}$, and then applies gradient descent to update them gradually. Each time, a subset $\mathcal{B}$ is sampled from $\mathcal{D}$, and a loss function computed according to the difference between prediction and labels:

$$
\mathcal{L}(\mathcal{B} ; \boldsymbol{\theta})=-\frac{1}{|\mathcal{B}|} \sum_{\left(\mathbf{x}_{n}, \mathbf{y}_{n}\right) \in \mathcal{B}} \mathbf{y}_{n}^{\top} \ln \mathbf{f}\left(\mathbf{x}_{n} ; \boldsymbol{\theta}\right)
$$


We can interpret this process as a heuristic way of searching over the high-dimensional parameter space defined by the network $\mathbf{f}(\mathbf{x} ; \boldsymbol{\theta})$. However, due to the complicated network design and limited dataset size, this training process often suffers over-fitting, i.e., a $\boldsymbol{\theta}$ is found to achieve a high accuracy on the training set, but the testing accuracy is still far below the training accuracy. This limits us from generalizing the trained model to unobserved testing data.

One way of softening supervision label signals is to perform teacher-student optimization. In this process, a teacher model $\mathbb{M}^{\mathrm{T}}: \mathbf{f}\left(\mathbf{x} ; \boldsymbol{\theta}^{\mathrm{T}}\right)$ is first trained in the same dataset using Eqn (1), and then used the model obtained in the last iteration (Hu et al. 2016) to train a student model $\mathbb{M}^{\mathrm{S}}: \mathbf{f}\left(\mathbf{x} ; \boldsymbol{\theta}^{\mathrm{S}}\right)$ using a mixture loss (Furlanello et al. 2018):

$$
\begin{aligned}
\mathcal{L}^{\mathrm{S}}\left(\mathcal{B} ; \boldsymbol{\theta}^{\mathrm{S}}\right)=-\frac{1}{|\mathcal{B}|} \sum_{\left(\mathbf{x}_{n}, \mathbf{y}_{n}\right) \in \mathcal{B}}\left\{\lambda \cdot \mathbf{y}_{n}^{\top} \ln \mathbf{f}\left(\mathbf{x}_{n} ; \boldsymbol{\theta}^{\mathrm{S}}\right)+\right. \\
\\
\left.(1-\lambda) \cdot \operatorname{KL}\left[\mathbf{f}\left(\mathbf{x}_{n} ; \boldsymbol{\theta}^{\mathrm{T}}\right) \| \mathbf{f}\left(\mathbf{x}_{n} ; \boldsymbol{\theta}^{\mathrm{S}}\right)\right]\right\} .
\end{aligned}
$$

This is to say, the teacher network provides $\mathbf{f}\left(\mathbf{x}_{n} ; \boldsymbol{\theta}^{\mathrm{T}}\right)$, a softened version of the one-hot vector $\mathbf{y}_{n}$, so that the student network can find a compromise between these two signals. In the next subsection, we will show how this formulation helps in training a better student.

A straightforward extension of teacher-student optimization is to allow a network to be optimized in generations. This requires training a patriarch model, denoted by $\mathbb{M}^{(0)}$, which is only supervised by the dataset. $M$ more generations follow, in which the $m$-th generation trains a student $\mathbb{M}^{(m)}$ with the supervision of a teacher $\mathbb{M}^{(m-1)}$. Most often (Furlanello et al. 2018), the recognition accuracy goes up in the first few generations, but starts to saturate and go down. We will analyze the reason in the following parts.

\section{Preserving Secondary Information: An Important Factor in Teacher-Student Optimization}

Previously, teacher-student optimization was mostly applied to distill knowledge from a larger network, so that it can be compressed into a smaller network with recognition accuracy largely preserved (Hinton, Vinyals, and Dean 2015), or applied to initializing a deeper network with pretrained weights from a shallower network (Romero et al. 2014)(Chen, Goodfellow, and Shlens 2015). As the first work to train an identical network in generations, (Furlanello et al. 2018) explained the benefit as a weighted balance between the ground-truth (one-hot) signal and the teacher signal, but it did not notice an important role of the teacher: suggesting class-level similarity.

To reveal this, we investigate network training of a bornagain process (Furlanello et al. 2018), with a 110-layer ResNet optimized on CIFAR100. In Table 1, we list the training and testing accuracies in each generation. Guided by softened distributions, the students achieve higher recognition performance than the patriarch. We hence ask a question: what is the key benefit of being trained by a softened label distribution? To answer it, we perform statistics on the class with second highest confidence score, and plot

\begin{tabular}{|l||r|r|r|r|r|r|}
\hline & Top-1 & Top-2 & Top-3 & Top-4 & Train & Test \\
\hline \hline Gen \#0 & 99.28 & 0.57 & 0.09 & 0.03 & 99.74 & 71.55 \\
\hline Gen \#1 & 98.68 & 1.00 & 0.18 & 0.06 & 99.63 & 71.41 \\
\hline Gen \#2 & 98.42 & 1.13 & 0.23 & 0.09 & 99.60 & 72.30 \\
\hline Gen \#3 & 98.33 & 1.19 & 0.24 & 0.09 & 99.62 & 72.26 \\
\hline Gen \#4 & 98.28 & 1.24 & 0.25 & 0.09 & 99.59 & 72.52 \\
\hline
\end{tabular}

Table 1: Confidence distribution (\%) on top-4 classes (individually determined for each training sample), obtained in a born-again process (with one patriarch and 5 more generations), training a 110-layer ResNet on CIFAR100. We also show training and testing accuracies $(\%)$ in the last columns.
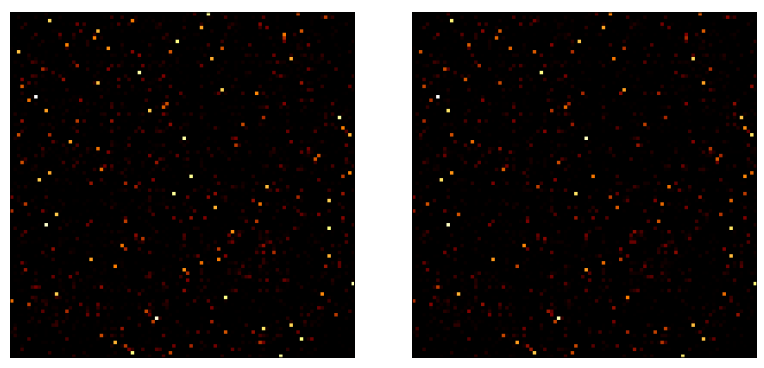

Figure 1: The $100 \times 100$ confusion matrices produced by the patriarch and the first student of a born-again process, training a 110-layer ResNet on CIFAR100. See Table 1 for quantitative numbers. The rows in these matrices indicate the ground-truth class, and the columns indicate the class with the second highest confidence score. The element $e_{i j}$ of the matrix is the frequency that a sample which belongs to class $i$ has the the second highest probability at class $j$. The color of a cell is closer to yellow when the corresponding value is larger.

the results as a confusion matrix in Figure 1. We find that a deep network is able to automatically learn semantically similar classes for each image individually ${ }^{1}$. We name it as secondary information, which corresponds to the primary information provided by supervision. In addition, we observe that these two confusion matrices are similar, indicating that the secondary information has been passed on from the teacher to the student. By taking these image-dependent information, the student network can avoid being fit to unnecessarily strict distributions and thus generalizes better. This motivates us to design a mechanism to measure the quality of secondary information and then try to construct better teacher signals.

\section{Towards High-Quality Secondary Information}

First, we note that the key to finding secondary information is to soften the output feature vector. We investigate three ways to achieve this goal. The first two methods follows two pieces of prior work named label smoothing regularization (LSR) (Szegedy et al. 2016) and confidence penalty

\footnotetext{
${ }^{1}$ For example, cat images are often considered similar to dog, but sometimes deer becomes the most similar class; automobile is most similar to truck in $60 \%$ of time, but in another $19 \%$ and $7 \%$ of time, it is most similar to ship and airplane, respectively.
} 
(CP) (Pereyra et al. 2017). Both of them added an extra term to the original cross-entropy loss, pushing the score distribution (after softmax) towards less peaked at the primary class. In LSR, the added term is the KL-divergence between the score distribution and the uniform distribution, while in $\mathrm{CP}$, it is the negative entropy gain. These two methods have a common drawback: they facilitate the confidence scores to distribute over all classes, regardless if these classes are visually similar to the training sample.

As the third option, we propose a more reasonable approach. Instead of computing an extra loss over all classes, we pick up a few classes which have been assigned with the highest confidence scores, and assume that these classes are more likely to be semantically similar to the input image. We set a fixed integer $K$ which stands for the number of semantically reasonable classes for each image, including the primary class ${ }^{2}$. Then, we compute the gap between the confidence scores of the primary class and other $K-1$ classes with highest scores:

$$
\begin{array}{r}
\mathcal{L}^{\mathrm{T}}\left(\mathcal{B} ; \boldsymbol{\theta}^{\mathrm{T}}\right)=\frac{1}{|\mathcal{B}|} \sum_{\left(\mathbf{x}_{n}, \mathbf{y}_{n}\right) \in \mathcal{B}}\left\{-\eta \cdot \mathbf{y}_{n}^{\top} \ln \mathbf{f}\left(\mathbf{x}_{n} ; \boldsymbol{\theta}^{\mathrm{T}}\right)+\right. \\
\left.(1-\eta) \cdot\left[f_{a_{1}}-\frac{1}{K-1} \sum_{k=2}^{K} f_{a_{k}}\right]\right\} .
\end{array}
$$

We name this method as top score difference (TSD), where $f_{a_{k}}^{\mathrm{T}}$ is short for the $k$-th largest element of $\mathbf{f}\left(\mathbf{x}_{n} ; \boldsymbol{\theta}^{\mathrm{T}}\right)$, and $\eta$ is a hyper-parameter controlling the balance between the ground-truth supervision and the score penalty term.

We evaluate these three options, as well as the baseline (using one-hot vectors, Eqn (1)), on their ability of preserving secondary information. To this end, we train a 110-layer ResNet on CIFAR100. Detailed settings can be found in the Experiments section. We perform four individual trainingin-generation processes, with the only difference lying in the patriarchs, i.e., four different options are applied. All the remaining generations follow Eqn (2) with $\lambda=0.6$. To maximally make fair comparison, we guarantee the same initialization weights for each model.

According to our conjecture, a good patriarch should preserve more secondary information, and thus is less discriminative in fine-level classes. We measure this factor at the class level. After these networks are trained, we analyze their behavior on both training and testing sets. For each image, the neural responses from the last residual block $(8 \times$ $8 \times 64)$ are average-pooled to obtain a 64 -dimensional vector. The training and testing sets of CIFAR100 has 50,000 and 10,000 images, which are uniformly distributed over 100 classes. These 100 classes are partitioned into 20 superclasses, with 5 fine-level classes in each superclass $\mathcal{S}_{j}$. We compute the mean vector for each class, denoted by $\mathbf{v}_{i}^{\mathrm{C}}$, $i=1,2, \ldots, 100$, and for each superclass, denoted by $\mathbf{v}_{j}^{\mathrm{S}}$, $j=1,2, \ldots, 20$, respectively.

Based on these, we compute two statistics within each superclass and among different superclasses, respectively. The

\footnotetext{
${ }^{2}$ Using a fixed $K$ may not be optimal, but it simplifies our approach and also works sufficiently well in either network training or transferring a trained network to other recognition tasks (see experiments).
}

\begin{tabular}{|l||r|r||r|r|r|}
\hline \multicolumn{1}{|c||}{} & \multicolumn{2}{c||}{ Training $^{\mathrm{C}}$} & \multicolumn{3}{c|}{ Testing } \\
\cline { 2 - 6 } & Dist $^{\mathrm{C}}$ & Dist $^{\mathrm{S}}$ & Dist $^{\mathrm{C}}$ & Dist $^{\mathrm{S}}$ & Best Acc \\
\hline \hline BL & 0.3813 & 0.4202 & 0.3234 & 0.3881 & $72.61 \%$ \\
\hline \hline LSR & 0.6182 & 0.5475 & 0.4875 & 0.4805 & $73.46 \%$ \\
\hline CP & 0.3829 & 0.4114 & 0.3267 & 0.3815 & $72.86 \%$ \\
\hline \hline TSD-0.6 & 0.3461 & $\mathbf{0 . 5 7 5 9}$ & 0.3153 & $\mathbf{0 . 5 3 4 9}$ & $\mathbf{7 3 . 7 2} \%$ \\
\hline TSD-0.7 & $\mathbf{0 . 3 4 3 3}$ & 0.5274 & $\mathbf{0 . 3 0 8 6}$ & 0.4894 & $73.18 \%$ \\
\hline TSD-0.8 & 0.3499 & 0.4782 & 0.3097 & 0.4449 & $73.39 \%$ \\
\hline
\end{tabular}

Table 2: Statistics on different patriarchs. The definitions of Dist $^{\mathrm{C}}$ and Dist ${ }^{\mathrm{S}}$ are provided in Eqn (4). BL is for baseline (as in (Furlanello et al. 2018)), LSR for label smoothing regularization, $\mathrm{CP}$ for confidence penalty, and TSD- $u(\eta)$ for top-score difference with a parameter $u(\eta)$. We also report the best testing accuracy throughout 10 generations, which reflect the potential of the patriarch model.

\begin{tabular}{|l||r|r|r|r|r|r|}
\hline & Gen\#0 & Gen\#1 & Gen\#2 & Gen\#3 & Gen\#4 & Gen\#5 \\
\hline \hline BAN & $99.28 \%$ & $98.68 \%$ & $98.42 \%$ & $98.33 \%$ & $98.28 \%$ & $97.76 \%$ \\
\hline LSR & $89.41 \%$ & $92.09 \%$ & $93.78 \%$ & $94.89 \%$ & $95.73 \%$ & $95.91 \%$ \\
\hline CP & $99.07 \%$ & $98.25 \%$ & $97.93 \%$ & $97.66 \%$ & $97.47 \%$ & $97.01 \%$ \\
\hline TSD-0.6 & $66.51 \%$ & $76.27 \%$ & $82.58 \%$ & $87.08 \%$ & $89.89 \%$ & $91.72 \%$ \\
\hline
\end{tabular}

Table 3: Top-1 scores of different generations trained with different patriarchs. The results are obtained by training a 110-layer ResNet on the CIFAR100 dateset. Gen \#0 represents the patriarch (Gen \#0 of BAN (Furlanello et al. 2018) is trained by one-hot vectors), and we also report the results of the first five students.

first one measures how much feature vector $\mathbf{v}_{i}^{\mathrm{C}}$ differs from the mean of its superclass, $\mathbf{v}_{j}^{\mathrm{S}}$ where $i \in \mathcal{S}_{j}$. Similarly, the second one measures the difference between each $\mathbf{v}_{j}^{\mathrm{S}}$ and the overall average vector $\mathbf{v}^{\mathrm{A}}=\frac{1}{20} \sum_{j} \mathbf{v}_{j}^{\mathrm{S}}$. Mathematically, these two metrics are:

$$
\begin{aligned}
\operatorname{Dist}^{\mathrm{C}} & =\frac{1}{100} \times \sum_{i=1}^{100} \arccos \frac{\left\langle\mathbf{v}_{i}^{\mathrm{C}}, \mathbf{v}_{j(i)}^{\mathrm{S}}\right\rangle}{\left\|\mathbf{v}_{i}^{\mathrm{C}}\right\| \cdot\left\|\mathbf{v}_{j(i)}^{\mathrm{S}}\right\|}, \\
\operatorname{Dist}^{\mathrm{S}} & =\frac{1}{20} \times \sum_{j=1}^{20} \arccos \frac{\left\langle\mathbf{v}_{j}^{\mathrm{S}}, \mathbf{v}^{\mathrm{A}}\right\rangle}{\left\|\mathbf{v}_{j}^{\mathrm{S}}\right\| \cdot\left\|\mathbf{v}^{\mathrm{A}}\right\|},
\end{aligned}
$$

where $j(i)$ denotes the superclass index for class $i$.

Results are summarized in Table 2. Compared to the baseline, LSR features are much more discriminative (both Dist $^{\mathrm{C}}$ and Dist ${ }^{\mathrm{S}}$ are much larger), while CP does not behave much differently. TSD-0.6 $(u(\eta)$ is an intuitive way of parameterizing $\eta$ - see the next part for details) best satisfies our assumption: increasing Dist $^{\mathrm{S}}$ so that coarse-level classification becomes better, meanwhile decreasing Dist ${ }^{\mathrm{C}}$ so that reasonable secondary information is preserved and learned by students. Using TSD- 0.7 or TSD- 0.8 does not heavily impact Dist ${ }^{\mathrm{C}}$, but causes Dist ${ }^{\mathrm{S}}$ to be much smaller. The best classification accuracy is obtained by TSD-0.6. In Table3, we show the trends of top-1 scores for comparison. In BAN and CP, the model's confidence is getting lower through generations, while in LSR and TSD-0.6, two "tolerant" patriarchs, the confidence is getting higher. 
Based on these observations, we can conclude that: in teacher-student optimization, the student learns best from a teacher that preserves reasonable secondary information. Hence, we suggest a framework that starts with a tolerant patriarch to optimize deep networks in generations.

\section{Details of Training in Generations}

We set Eqn (3) to be the loss function to train the patriarch model $\mathbb{M}^{(0)}$. Mathematically, to minimize Eqn (3), $\mathbf{f}\left(\mathbf{x}_{n} ; \boldsymbol{\theta}^{\mathrm{T}}\right)$ shall satisfy $0<f_{a_{1}}^{\mathrm{T}}<1, f_{a_{2}}^{\mathrm{T}}+\ldots+f_{a_{K}}^{\mathrm{T}}=$ $1-f_{a_{1}}^{\mathrm{T}}$, and all other entries are 0 . We can derive the optimal $f_{a_{1}}^{\mathrm{T}} \doteq u(\eta)=\min \left\{\frac{\eta}{1-\eta} \cdot \frac{K-1}{K}, 1\right\}$, which is a monotonically increasing function with respect to $\eta$. Therefore, it is equivalent to consider $u(\eta)$ instead of $\eta$. In experiments, we shall see that a deeper network often requires a larger $u(\eta)$, or equivalently a larger $\eta$, in order to get better trained.

Two side notes are made on Eqn (3) and the hyperparameter $K$. First, our formulation does not guarantee the primary class $a_{1}$ corresponds to the true class. But as we shall see in experiments, after a sufficient number of training epochs, the training accuracy is always close to $100 \%$. Second, $K$ is often difficult to estimate, and may vary among different primary classes. In practice, we fix $K=5$ for simplicity $^{3}$.

Then, $M$ generations follow the patriarch model. At the $m$-th generation, $\mathbb{M}^{(m)}$ learns from $\mathbb{M}^{(m-1)}$ using Eqn (2), with models $\mathbf{f}\left(\mathbf{x}_{n} ; \boldsymbol{\theta}^{\mathrm{T}}\right)$ and $\mathbf{f}\left(\mathbf{x}_{n} ; \boldsymbol{\theta}^{\mathrm{S}}\right)$ replaced by $\mathbf{f}\left(\mathbf{x}_{n} ; \boldsymbol{\theta}^{(m-1)}\right)$ and $\mathbf{f}\left(\mathbf{x}_{n} ; \boldsymbol{\theta}^{(m)}\right)$, respectively. Similarly, the optimal $\mathbf{f}\left(\mathbf{x}_{n} ; \boldsymbol{\theta}^{(m)}\right)$ to minimize $\mathcal{L}^{(m)}$ shall satisfy $0<f_{a_{1}}^{(m)}<1, f_{a_{2}}^{(m)}+\ldots+f_{a_{K}}^{(m)}=\left(1-f_{a_{1}}^{(m)}\right)$, and all other entries are 0 . So, we have $f_{a_{1}}^{(m)} \doteq w\left(\lambda, \mathbf{f}^{(m-1)}\right)$ which is monotonically increasing with respect to $\lambda$.

In summary, the entire optimization process is parameterized by $K, \eta$ and $\lambda$. We fix $K=5$ and use $u(\eta)$ to equivalently replace $\eta$, so that each process is denoted by $\mathfrak{D}(u(\eta), \lambda)$. As special cases of our approach, the conventional network optimization process can be abbreviated as $\mathfrak{D}(1.0,0.0)$, and training a born-again network (Furlanello et al. 2018) corresponds to $\mathfrak{D}(1.0,0.5)$.

Last but not least, given that the trained model $\mathbf{f}(\cdot ; \boldsymbol{\theta})$ has a sufficient ability of fitting data, we have $f_{a_{1}}^{(m)} \rightarrow 1$ as $m \rightarrow+\infty$, regardless of $u(\eta)$ and $\lambda$. Therefore, the secondary information in teacher signals is gradually weakened, and the effect of optimizing Eqn (2) is more and more similar to optimizing Eqn (1). This implies that recognition accuracy may saturate and start to descend after a few generations (see Figure 2 for experimental results).

\footnotetext{
${ }^{3}$ This fits CIFAR100 well, because it contains 20 coarse groups and each of them has 5 finer-level classes. This setting also works well in ILSVRC2012, although there are different numbers of semantically similar classes for each class. We transfer the trained models on ILSVRC2012 to other recognition tasks to reveal the generalization properties. See experiments for details.
}

\section{Experiments}

\section{The CIFAR100 Dataset}

\section{- Setting and Baselines}

We first evaluate our approach on the CIFAR100 dataset (Krizhevsky and Hinton 2009), which contains 60,000 tiny RGB images of a spatial resolution of $32 \times 32$. These images are split into a training set of 50,000 samples and a testing set of 10,000 samples. Both training and testing images are uniformly distributed over 100 classes. We do not perform experiments on the CIFAR10 dataset because it does not contain fine-level classes, so that teacherstudent optimization does not bring significant benefits (this was also observed in (Furlanello et al. 2018)).

We experiment on deep residual networks (He et al. 2016) with 20, 56 and 110 layers, and densely connected convolutional networks (DenseNets) (Huang et al. 2017b) with 100 and 190 layers. We follow the conventions to train these networks from scratch. We use the standard Stochastic Gradient Descent (SGD) with a weight decay of 0.0001 and a Nesterov momentum of 0.9 . In the ResNets, we train the network for 164 epochs with mini-batch size of 128 . The base learning rate is 0.1 , and is divided by 10 after 82 and 123 epochs. In the DenseNets, we train the network for 300 epochs with a mini-batch size of 64 . The base learning rate is 0.1 , and is divided by 10 after 150 and 225 epochs. In the training process, standard data-augmentation is used, i.e., each image is padded with a 4-pixel margin on each of the four sides. In the enlarged $40 \times 40$ image, a subregion with $32 \times 32$ pixels is randomly cropped and flipped with a probability of 0.5 . No augmentation is used at the testing stage.

\section{- Results}

We first evaluate the performance with respect to different hyper-parameters, namely, different parameterized processes $\mathfrak{D}(u(\eta), \lambda)$. We fix $K=5$ and $u(\eta)=0.6$, and diagnose the impact of $\lambda$ on deep residual networks (He et al. 2016) with different numbers of layers. We also evaluate the born-again networks (Furlanello et al. 2018) which corresponds to $\mathfrak{D}(1.0,0.5)$.

Results on deep ResNets are summarized in Figure 2. We can observe several important properties of our algorithm. First, a strict teacher (i.e., the born-again network (Furlanello et al. 2018), $\mathfrak{D}(1.0,0.6)$ ) is inferior to a tolerant teacher $(e . g ., \mathfrak{D}(0.6,0.6))$. Although the latter often starts with a lower accuracy of the patriarch model, it has the ability of gradually and persistently growing up and outperforming the baseline after 1-3 generations. Meanwhile, recognition accuracy often saturates after a few generations, because eventually the teacher signal will converge to the points that are dominated by the primary classes (i.e., $f_{a_{1}}^{(m)} \rightarrow 1$ ), and the teacher will become strict again. However, the saturated accuracy is still much higher than the baseline, which demonstrates the reliability of our approach, i.e., even if we cannot terminate at the best generation, we can still surpass the baseline.

In DenseNets with 100 and 190 layers, we report both single-model and model-ensemble results in Table 4 . We

\footnotetext{
${ }^{5}$ github.com/bearpaw/pytorch-classification
} 



Figure 2: Classification accuracy (\%) on CIFAR100, produced by different training-in-generation processes. The baseline approach (single generation) corresponds to $\mathfrak{D}(1.0,0.0)$, and $\mathfrak{D}(1.0,0.5)$ and $\mathfrak{D}(1.0,0.6)$ are born-again networks. LSR-0.6 and CP-0.6 indicate replacing the patriarch model with label smoothing regularization and confidence penalty, and use $\lambda=0.6$ in generations. All three plots share the same legend (shown in the first plot).

\begin{tabular}{|c|c|c|c|c|c|c|c|}
\hline & Gen \#0 & Gen \#1 & Gen \#2 & Gen \#3 & Gen \#4 & \multicolumn{2}{|c|}{ Gen \#5 } \\
\hline Baseline (100 lic & $22.20(22.89)$ &  & - & - & - & \multirow{2}{*}{\multicolumn{2}{|c|}{$\begin{array}{r}- \\
21.27(21.61)\end{array}$}} \\
\hline $\mathfrak{D}(0.6,0.6)$ & $23.96(25.00)$ & $21.29(21.34)$ & $\mathbf{2 0 . 5 1}(21.59)$ & $20.83(20.99)$ & $21.01(21.53)$ & & \\
\hline +Ensemble & - & 20.20 & 18.38 & 17.79 & 17.37 & \multirow{2}{*}{\multicolumn{2}{|c|}{$\frac{\mathbf{1 7 . 2 5}}{21.87(22.28)}$}} \\
\hline $\mathfrak{D}(0.7,0.6)$ & $22.98(23.43)$ & $21.24(21.50)$ & $21.48(21.80)$ & $\mathbf{2 0 . 9 4}(21.47)$ & $21.51(21.69)$ & & \\
\hline +Ensemble & - & 19.63 & 18.83 & 17.70 & 17.56 & & 17.23 \\
\hline Baseline (190 lay & $17.22(17.62)$ & 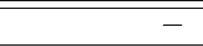 & - & - & - & & - \\
\hline $\mathfrak{D}(0.6,0.6)$ & $18.87(19.40)$ & $17.42(17.99)$ & $17.26(18.00)$ & $17.13(17.52)$ & $17.24(17.75)$ & \multicolumn{2}{|c|}{$\mathbf{1 7 . 0 1}(17.22)$} \\
\hline +Ensemble & - & 16.83 & 15.94 & 15.43 & 15.18 & \multirow{2}{*}{\multicolumn{2}{|c|}{$\begin{array}{r}15.21 \\
17.24(17.41)\end{array}$}} \\
\hline $\mathfrak{D}(0.7,0.6)$ & $18.63(19.12)$ & $17.44(17.78)$ & $\mathbf{1 6 . 7 2}(17.21)$ & $16.89(16.98)$ & $17.39(17.71)$ & & \\
\hline +Ensemble & - & 16.37 & 15.20 & 15.11 & 14.93 & \multicolumn{2}{|r|}{14.47} \\
\hline \multicolumn{2}{|l|}{ (Zhang et al. 2017b) } & \multicolumn{2}{|c|}{ (Huang et al. 2017a) } & "17.40 & \multicolumn{2}{|c|}{ (Han, Kim, and Kim 2017) } & 17.01 \\
\hline (Zhang et al. 2017a) & 16.80 & (Gastaldi 201 & & 15.85 & nello et al. 2018 & & 14.90 \\
\hline
\end{tabular}

Table 4: Classification error rates (\%) by different models on CIFAR100. All networks in each group have the same depth. Gen \#0 stands for the patriarch. We use a GitHub repository ${ }^{5}$ as our baseline, and our re-implementation results are comparable to those reported originally (22.80\% for DenseNet-100 and $17.17 \%$ for DenseNet-190). Following conventions, we report accuracies from both the best epoch and the last epoch (numbers in parentheses), and all listed competitors reported the best epoch. We perform model ensemble on the last epochs of all generations.

evaluate $\mathfrak{D}(0.6,0.6)$ and $\mathfrak{D}(0.7,0.6)$, and observe the same phenomena as in ResNet experiments. In particular, in DenseNet-100, our single-model accuracy is 1\%$2 \%$ higher, and our 5-model ensemble accuracy is more than $5 \%$ higher, even getting close to a single DenseNet190 model. Considering DenseNet-190 requires around $30 \times$ FLOPs of DenseNet-100, this is quite an efficient method to achieve high classification accuracy. In DenseNet-190, our results are competitive among the state-of-the-arts. Note that (Zhang et al. 2017a) and (Gastaldi 2017) applied complicated data augmentation approaches to achieve high accuracy, but we found a different way, which is to improve the optimization algorithm.

\section{The ILSVRC2012 Dataset}

\section{- Setting and Baselines}

With the knowledge and parameters learned from the CIFAR100 experiments, we now investigate the ILSVRC2012 dataset (Russakovsky et al. 2015), a popular subset of the ImageNet database (Deng et al. 2009). There are 1,000 classes in total. The training set and testing set contains $1.3 \mathrm{M}$ and $50 \mathrm{~K}$ high-resolution images, with each class having approximately the same number of training images and exactly the same number of testing images.

We set the 18-layer residual network (He et al. 2016) as our baseline. All networks are trained from scratch. We follow (Hu, Shen, and Sun 2018) in configuring the following parameters. Standard Stochastic Gradient Descent (SGD) with a weight decay of 0.0001 and a Nesterov momentum of 0.9 is used. There are a total of 100 epochs in the training process, and the mini-batch size is 1024 . The learning rate starts with 0.1 , and is divided by 10 after 30,60 and 90 epochs. In the training process, we apply a series of dataaugmentation techniques, including rescaling and cropping the image, randomly mirroring and rotating (slightly) the image, changing its aspect ratio and performing pixel jittering. In the testing stage, the standard single-center-crop is used on each image.

We inherit the best parameters learned from CIFAR100 experiments to ILSVRC2012 (the costly computation avoids 


\begin{tabular}{|c|c|c|c|c|c|c|c|c|c|c|c|c|}
\hline & \multicolumn{2}{|c|}{ Gen \#0 } & \multicolumn{2}{|c|}{ Gen \#1 } & \multicolumn{2}{|c|}{ Gen \#2 } & \multicolumn{2}{|c|}{ Gen \#3 } & \multicolumn{2}{|c|}{ Gen \#4 } & \multicolumn{2}{|c|}{ Gen \#5 } \\
\hline Baseline & 30.50 & 11.07 & - & - & - & - & - & - & - & - & - & - \\
\hline $\mathfrak{D}(0.6,0.6)$ & 32.52 & 11.23 & 30.28 & 10.23 & 30.12 & 10.15 & 29.92 & 10.25 & 29.77 & 10.19 & 29.60 & 10.11 \\
\hline +Ensemble & - & - & 30.01 & 9.98 & 28.94 & 9.53 & 28.51 & 9.36 & 28.23 & 9.28 & 28.08 & 9.23 \\
\hline
\end{tabular}

Table 5: Classification error rates (top-1 and top-5, \%) by different ResNet-18 models on ILSVRC2012.

us from tuning the hyper-parameters). We use $\mathfrak{D}(0.6,0.6)$, as the basic network (18 layers) is not very deep).

\section{- Results}

Following CIFAR100 experiments, we set $K=5, u(\eta)=$ 0.6 and $\lambda=0.6$. Results are summarized in Table 5. One can observe very similar results as in the previous experiments, i.e., we start with a worse patriarch ${ }^{6}$, enjoy gradual and persistent improvement from generation to generation, and achieve saturation after several generations.

Limited by computational resources, we did not evaluate deeper networks. Although the performance of ResNet-18 is not directly comparable to deeper networks (e.g., ResNets with 50, 101, 152 layers or DenseNet with 264 layers), our approach achieves a larger accuracy gain (0.90\% top1 and $0.96 \%$ top-5) than other two light-weighted modules on ResNet-18, namely Squeeze-and-Excitation (SE) (Hu, Shen, and Sun 2018) (0.72\% top-1 and 0.80\% top-5) and Second-Order Response Transform (SORT) (Wang et al. 2017) ( $0.55 \%$ top- 1 and $0.27 \%$ top- 5$)$. Compared to them, our approach does not require any additional computation at the testing stage, although the training stage is longer.

\section{- Transfer Experiments}

Finally, we transfer the trained models to feature extraction. We consider two popular datasets, namely, Caltech256 (Griffin, Holub, and Perona 2007) and MIT Indoor67 (Quattoni and Torralba 2009) for generic object classification and indoor scene classification, respectively. We follow the conventional settings, i.e., extracting neural responses from the penultimate layer of the 18-layer ResNet, and training a linear SVM to classify these 512-dimensional vectors. We use the same training/testing split for all models, with 60 and 80 training images per class for Caltech256 and MIT Indoor-67, respectively.

Results are summarized in Table 6. We can find that, besides achieving better recognition accuracy on ILSVRC2012, the networks obtained by our approach indeed produce higher-quality transferrable features. These transfer experiments also verify that setting $K=5$ generalizes well to other recognition tasks, i.e., the intrinsic benefit comes from the secondary information, and our approach does not rely on a specific $K$. We believe these models also perform better in other transfer learning scenarios, e.g., being fine-tuned on the PascalVOC or MS-COCO datasets.

\footnotetext{
${ }^{6}$ It is interesting yet expected that the top- 1 accuracy of the patriarch is $1.98 \%$ lower than the baseline, but the top-5 accuracy is merely $0.16 \%$ lower. This is because setting $K=5$ hardly impacts top- 5 classification.
}

\begin{tabular}{|l||r|r||r|r|}
\hline \multicolumn{1}{|c||}{} & \multicolumn{2}{c||}{ ILSVRC2012 } & \multicolumn{2}{c|}{ Transfer } \\
\cline { 2 - 5 } & Top-1 & Top-5 & C256 & I67 \\
\hline \hline Baseline & 69.50 & 88.93 & 75.94 & 62.92 \\
\hline \hline Gen \#0 & 67.48 & 88.77 & 75.15 & 62.97 \\
\hline Gen \#1 & 69.72 & 89.77 & 76.83 & 62.75 \\
\hline Gen \#2 & 69.88 & 89.85 & 77.28 & $\mathbf{6 5 . 7 2}$ \\
\hline Gen \#3 & 70.08 & 89.75 & 77.65 & 63.75 \\
\hline Gen \#4 & 70.23 & 89.81 & 77.00 & 63.81 \\
\hline Gen \#5 & $\mathbf{7 0 . 4 0}$ & $\mathbf{8 9 . 8 9}$ & $\mathbf{7 7 . 8 3}$ & 63.94 \\
\hline
\end{tabular}

Table 6: Classification accuracies (\%) by different models on Caltech256 (C256) and MIT Indoor-67 (I67 datasets. Top-1 and top-5 classification accuracies (\%) on ILSVRC2012 are also listed for reference.

\section{Conclusions}

In this work, we study the problem of optimizing deep networks in generations. This problem is meaningful, because it allows us to explore network optimization in depth; it is also useful, as we obtain better networks with the same architecture - although training time becomes longer, testing time (including in transfer experiments) remains unchanged.

Based on the existing works, we provide a new viewpoint that teacher models should preserve secondary information, so that the students become stronger. We quantify these information, and empirically verify its impact in image classification. We train some standard networks on image classification datasets, and then transfer them to other recognition tasks. Our approach surpasses the single-generation and multi-generation baselines in every single case.

This research votes for the viewpoint that network optimization is far from perfect at the current status. In the future, we will investigate a more generalized model, including using a variable function at each generation and allowing $K$ to vary from case to case. In addition, we will consider a temperature term in Eqn (2) to adjust the KL-divergence. Both are expected to achieve better optimization results.

Acknowledgements This paper is supported by NSF award CCF-1317376 and ONR award N00014-15-1-2356.

\section{References}

Akata, Z.; Perronnin, F.; Harchaoui, Z.; and Schmid, C. 2016. Label-embedding for image classification. TPAMI 38(7):14251438 .

Chen, L. C.; Papandreou, G.; Kokkinos, I.; Murphy, K.; and Yuille, A. L. 2016. Deeplab: Semantic image segmentation with deep convolutional nets, atrous convolution, and fully connected crfs. In $I C L R$. 
Chen, T.; Goodfellow, I.; and Shlens, J. 2015. Net2net: Accelerating learning via knowledge transfer. arXiv preprint arXiv:1511.05641.

Deng, J.; Dong, W.; Socher, R.; Li, L.; Li, K.; and Fei-Fei, L. 2009. Imagenet: A large-scale hierarchical image database. In $C V P R$.

Deng, J.; Berg, A. C.; Li, K.; and Fei-Fei, L. 2010. What does classifying more than 10,000 image categories tell us? In ECCV.

Donahue, J.; Jia, Y.; Vinyals, O.; Hoffman, J.; Zhang, N.; Tzeng, E.; and Darrell, T. 2014. Decaf: A deep convolutional activation feature for generic visual recognition. In ICML.

Furlanello, T.; Lipton, Z. C.; Itti, L.; and Anandkumar, A. 2018. Born again neural networks. In ICML.

Gastaldi, X. 2017. Shake-shake regularization. arXiv preprint arXiv:1705.07485.

Girshick, R.; Donahue, J.; Darrell, T.; and Malik, J. 2014. Rich feature hierarchies for accurate object detection and semantic segmentation. In $C V P R$.

Girshick, R. 2015. Fast r-cnn. In CVPR.

Griffin, G.; Holub, A.; and Perona, P. 2007. Caltech-256 object category dataset.

Han, D.; Kim, J.; and Kim, J. 2017. Deep pyramidal residual networks. In $C V P R$.

He, K.; Zhang, X.; Ren, S.; and Sun, J. 2016. Deep residual learning for image recognition. In CVPR.

Hinton, G.; Vinyals, O.; and Dean, J. 2015. Distilling the knowledge in a neural network. arXiv preprint arXiv:1503.02531.

Hu, Z.; Ma, X.; Liu, Z.; Hovy, E.; and Xing, E. 2016. Harnessing deep neural networks with logic rules. In $A C L$.

Hu, J.; Shen, L.; and Sun, G. 2018. Squeeze-and-excitation networks. In $C V P R$.

Huang, G.; Li, Y.; Pleiss, G.; Liu, Z.; Hopcroft, J. E.; and Weinberger, K. Q. 2017a. Snapshot ensembles: Train 1, get $\mathrm{m}$ for free. In ICLR.

Huang, G.; Liu, Z.; Weinberger, K. Q.; and van der Maaten, L. 2017b. Densely connected convolutional networks. In CVPR.

Ioffe, S., and Szegedy, C. 2015. Batch normalization: Accelerating deep network training by reducing internal covariate shift. In ICML.

Krizhevsky, A., and Hinton, G. 2009. Learning multiple layers of features from tiny images.

Krizhevsky, A.; Sutskever, I.; and Hinton, G. E. 2012. Imagenet classification with deep convolutional neural networks. In NIPS.

Long, J.; Shelhamer, E.; and Darrell, T. 2015. Fully convolutional networks for semantic segmentation. In $C V P R$.

Nair, V., and Hinton, G. E. 2010. Rectified linear units improve restricted boltzmann machines. In ICML.

Newell, A.; Yang, K.; and Deng, J. 2016. Stacked hourglass networks for human pose estimation. In $E C C V$.

Pereyra, G.; Tucker, G.; Chorowski, J.; Kaiser, Ł.; and Hinton, G. 2017. Regularizing neural networks by penalizing confident output distributions. arXiv preprint arXiv:1701.06548.

Perronnin, F.; Sanchez, J.; and Mensink, T. 2010. Improving the fisher kernel for large-scale image classification. In ECCV.

Quattoni, A., and Torralba, A. 2009. Recognizing indoor scenes. In $C V P R$.

Razavian, A. S.; Azizpour, H.; Sullivan, J.; and Carlsson, S. 2014. Cnn features off-the-shelf: an astounding baseline for recognition. In Computer Vision and Pattern Recognition.
Ren, S.; He, K.; Girshick, R.; and Sun, J. 2015. Faster r-cnn: Towards real-time object detection with region proposal networks. In NIPS.

Romero, A.; Ballas, N.; Kahou, S. E.; Chassang, A.; Gatta, C.; and Bengio, Y. 2014. Fitnets: Hints for thin deep nets. arXiv preprint arXiv:1412.6550.

Russakovsky, O.; Deng, J.; Su, H.; Krause, J.; Satheesh, S.; Ma, S.; Huang, Z.; Karpathy, A.; Khosla, A.; Bernstein, M.; et al. 2015. Imagenet large scale visual recognition challenge. IJCV 115(3):211252.

Simonyan, K., and Zisserman, A. 2015. Very deep convolutional networks for large-scale image recognition. In ICLR.

Srivastava, N.; Hinton, G. E.; Krizhevsky, A.; Sutskever, I.; and Salakhutdinov, R. 2014. Dropout: A simple way to prevent neural networks from overfitting. JMLR 15(1):1929-1958.

Szegedy, C.; Liu, W.; Jia, Y.; Sermanet, P.; Reed, S.; Anguelov, D.; Erhan, D.; Vanhoucke, V.; Rabinovich, A.; et al. 2015. Going deeper with convolutions. In $C V P R$.

Szegedy, C.; Vanhoucke, V.; Ioffe, S.; Shlens, J.; and Wojna, Z. 2016. Rethinking the inception architecture for computer vision. In Computer Vision and Pattern Recognition.

Tarvainen, A., and Valpola, H. 2017. Mean teachers are better role models: Weight-averaged consistency targets improve semisupervised deep learning results. In NIPS.

Verma, N.; Mahajan, D.; Sellamanickam, S.; and Nair, V. 2012. Learning hierarchical similarity metrics. In $C V P R$.

Wang, J.; Leung, T.; Rosenberg, C.; Wang, J.; Philbin, J.; Chen, B.; $\mathrm{Wu}, \mathrm{Y}$; et al. 2014. Learning fine-grained image similarity with deep ranking. In $C V P R$.

Wang, Y.; Xie, L.; Liu, C.; Qiao, S.; Zhang, Y.; Zhang, W.; Tian, Q.; and Yuille, A. 2017. Sort: Second-order response transform for visual recognition. In $I C C V$.

Wu, C.; Tygert, M.; and LeCun, Y. 2017. Hierarchical loss for classification. arXiv preprint arXiv:1709.01062.

Xie, S., and Tu, Z. 2015. Holistically-nested edge detection. In ICCV.

Xie, L., and Yuille, A. 2017. Genetic cnn. In ICCV.

Yim, J.; Joo, D.; Bae, J.; and Kim, J. 2017. A gift from knowledge distillation: Fast optimization, network minimization and transfer learning. In $C V P R$.

Zhang, H.; Cisse, M.; Dauphin, Y. N.; and Lopez-Paz, D. 2017a. mixup: Beyond empirical risk minimization. arXiv preprint arXiv:1710.09412.

Zhang, T.; Qi, G. J.; Xiao, B.; and Wang, J. 2017b. Interleaved group convolutions. In CVPR.

Zhang, Y.; Xiang, T.; Hospedales, T. M.; and Lu, H. 2017c. Deep mutual learning. arXiv preprint arXiv:1706.00384.

Zhang, C.; Cheng, J.; and Tian, Q. 2018. Image-level classification by hierarchical structure learning with visual and semantic similarities. Information Sciences 422:271-281.

Zoph, B., and Le, Q. V. 2017. Neural architecture search with reinforcement learning. In ICLR. 